\title{
L'effetto delle operazioni di fusione e acquisizione sui profitti degli azionisti nell'industria farmaceutica
}

\author{
Grant H. Skrepnek, Kenneth A. Lawson
}

\author{
Articolo tratto dal Joumal of Research in Pharmaceutical Economics, Vol. Il (1) 2001 E 2001 by The \\ Haworth Press, Inc.
}

\begin{abstract}
The purpose of this study was to quantify the excess return in shareholder security prices following initial announcements of successful horizontal pharmaceutical mergers which occurred between 1985 and 1996. An event study methodology was used to study a sample of 26 fim1s involved in 14 mergers wherein transaction costs exceeded $\$ 500$ million.

Overall, results of the study were consistent with prior research, indicating that target-firm shareholders received a majority oft he short-term merger-related wealth effects in comparison to those received by bidder- firm shareholders.
\end{abstract}

\section{INTRODUZIONE}

Le aziende americane, per aumentare la forza corporativa, si sono impegnate in operazioni di fusione e acquisizione (M \& A) fin dal 1800.

L'onda più recente di fusioni, iniziata a metà degli anni ' 80 , è stata caratterizzata da un'attività $\mathrm{M} \& \mathrm{~A}$ molto sostenuta all'interno dell'industria farmaceutica. Tra il 1985 e il 1996, le fusioni di società nell'industria farmaceutica hanno raggiunto livelli sostenuti; le industrie farmaceutiche, di materiale e di attrezzatura medica statunitensi si sono posizionate nei primi 10 posti tra 50 industrie presenti negli annunci di fusioni apparsi in questi anni (1). L'impatto delle recenti fusioni di case farmaceutiche è risultato in una ristrutturazione dell'industria e i vantaggi di alcune di queste fusioni sono stati messi in discussione (2). Molti studi empirici sulle operazioni M \&A sono pubblicati in letteratura. Sebbene i primi lavori si concentrassero prevalentemente sulle basi teoriche delle fusioni, buona parte della ricerca condotta dalla metà degli anni ' 60 in poi ha cercato di determinare gli effetti economici delle operazioni M \& A. I risultati di questi studi hanno mostrato in maniera uniforme che le società acquirenti spesso subiscono solo lievi perdite o lievi guadagni, mentre le aziende acquisite hanno tipicamente beneficiato di rendimenti ampiamente positivi in seguito agli annunci di fusione coronata da successo. Jensen e Ruback hanno riassunto la maggior parte dei primi studi empirici riguardanti gli effetti economici e i guadagni degli azionisti associati alle attività di controllo delle aziende (3). I risultati di oltre 40 studi hanno indicato che le operazioni di acquisizione (via merger, tender offers, proxy contest) possono creare effetti economici positivi per gli azionisti, benché questi guadagni non siano risultati equamente distribuiti tra le aziende acquirenti e quelle acquisite. I rendimenti relativi al periodo compreso tra il primo annuncio

\footnotetext{
${ }^{*}$ Grant H. Skrepnek, R.Ph., M.S., is a doctoral candidate and Kenneth A. Lawson, Ph.D., is Associate Professo/; both in the Pharmacy Practice and Administration Division, College of Pharmacy, University ofTexas, Austin, TX 78712
} 
pubblico e il completamento della fusione sono stati del $-1,77 \%$ (range $=-7,22 \%-0,10 \%, \mathrm{~N}=256$ ) per gli acquirenti; le azioni delle aziende acquisite hanno ottenuto rendimenti del 20,15\% (range 15,50\% -33,96\%, $\mathrm{N}=282)$. Per i periodi compresi tra il giorno precedente l'annuncio e il giorno dell'annuncio $(-1,0)$ e tra il giorno precedente ed il giorno successivo all'annuncio $(-1,+1)$, gli acquirenti hanno ottenuto rendimenti extra compresi tra - 1,09\% e 0,07\% ( $\mathrm{N}=358)$, mentre le società acquisite hanno ottenuto risultati compresi tra il $6,24 \%$ e il $13,41 \%(\mathrm{~N}=339)$. La redditività associata agli annunci di fusione durante i periodi compresi tra $(-20,10)$ e $(-30,0)$ è situata tra lo $0,20 \%$ e il $3,48 \%$ per gli offerenti con successo $(\mathrm{N}=784)$ e tra $i 113,30 \%$ e i121, 78\% per le società acquisite $(\mathrm{N}=457)$. Anche Jarrell, Brickley e Netter hanno rianalizzato diversi studi empirici riguardanti il guadagno degli azionisti e le operazioni M\&A. Questi autori hanno ottenuto risultati concordanti con quelli presentati da Jensen e Ruback (4). Jarrel e Poulsen, nella loro analisi sulle tender offers tra il 1963 ed il 1986, hanno riportato che i rendimenti per le aziende acquirenti sono state del 1,29\% $(\mathrm{t}=2,35, \mathrm{~N}=462)$ durante il periodo $(-20,+10)$; le società acquisite nello stesso periodo hanno ottenuto il $28,99 \%(\mathrm{t}=30,50, \mathrm{~N}=526)(5)$.

Sebbene esista un considerevole volume di letteratura riguardo le fusioni farmaceutiche, è stata condotta relativamente poca ricerca empirica mirata a stabilire gli effetti di questa attività di fusione dell'industria sulla ricchezza degli azionisti. Lo scopo del presente studio è stato quello di quantificare le variazioni anomale di rendimento nel corso delle azioni associate a grandi fusioni orizzontali coronate da successo nell'ambito dell'industria farmaceutica tra gli anni 1985 e 1996.

\section{METODOLOGIA DELLA RICERCA}

Gran parte della recente letteratura sulle operazioni M\&A ha utilizzato una metodologia di studio dell'evento mediante la quale i rendimenti delle azioni sono calcolate sulla base del profitto atteso per quel dato titolo in quel determinato periodo. Con tale metodologia, i guadagni attesi, calcolati mediante un modello finanziario che elimini l'influenza di fattori estranei e generali del mercato e di altri fattori sistematici, vengono confrontati con il guadagno effettivo. Gli effetti economici delle operazioni di acquisizione sono spesso riportati sotto forma di ritorni anormali, cioè la differenza tra il guadagno effettivo e quello atteso dei titoli. La metodologia di studio dell'evento usata nel presente studio si è basata sulle raccomandazioni apparse negli studi di simulazione di Brown e Warner e Dyckman, Philbrick, e Stephan oltre che nei reviews di Bowman, Peterson, Henderson, Strong, e MacKinlay (6- 13).

Il campione di fusioni utilizzato in questo studio è stato selezionato in base a quattro criteri di inclusione: (1) I costi della transazione per una data fusione devono aver superato i 500 milioni di dollari, (2) le fusioni devono essersi concluse con successo tra il 1985 e il 1996, (3) le società acquisite devono essere state società farmaceutiche e le società acquirenti devono essere state aziende farmaceutiche o gruppi chimici, e (4) le informazioni sui valori giornalieri dei titoli devono essere riportate negli archivi del Center for Research in Security Price (CRSP) compilati dall'Università di Chicago (cioè le azioni corporative devono essere state attivamente scambiate sui mercati NYSE,AMEX o NASDAQ).

Inizialmente, mediante una revisione della cronaca finanziaria stampata dal 1985 al 1996, sono state identificate circa 30 fusioni $(1,14)$. Tra queste, 14 soddisfacevano tutti i criteri di inclusione dello studio. Per alcune delle fusioni inizialmente identificate non vi erano dati disponibili negli archivi delle maggiori borse statunitensi (la fusione SandozAG/Ciba-Geigy AG del 1996), altre fusioni sono state scartate perché non di carattere orizzontale. La tabella 1 riassume i dati relativi al giorno dell'annuncio, alle società acquirenti e acquisite, e i costi della transazione per ogni fusione compresa nello studio.

Sono state analizzate le modificazioni del prezzo delle azioni nel periodo dell'annuncio iniziale di una fusione o di un'acquisizione. In particolare, il giorno-evento (cioè il giorno 0 ) dello studio è stato definito come il giorno in cui nella stampa finanziaria è comparsa la divulgazione pubblica delle trattative di fusione tra i contraenti finali. Sulla base di metodologie precedenti, si è assunto che il giorno del primo annuncio 
formale di trattative di fusione comparso nella stampa finanziaria corrispondesse alla data in cui i mercati finanziari abbiano reagito all'informazione. Le date dei primi annunci di fusione ed acquisizione sono state individuate mediante una ricerca con Lexis? - Nexis? , limitata alle fusioni di produttori farmaceutici. Per gli annunci non rilevati dalla ricerca Lexis- Nexis, è stato consultato il Dow Jones News Service? per ottenere la data dell'annuncio iniziale.

\begin{tabular}{|c|c|c|c|}
\hline $\begin{array}{l}\text { Giorno di } \\
\text { annuncio }\end{array}$ & Società acquirenti & Società acquisite & $\begin{array}{c}\text { Costi di } \\
\text { transazione }+\end{array}$ \\
\hline $03 / 18 / 96$ & Elan Corp. PLC & Athena Neurosciences Inc. & $\$ 640$ \\
\hline $08 / 19 / 95$ & Upjohn Co. & Pharmacia $\mathrm{AB}$ & $\$ 6,000$ \\
\hline $08 / 18 / 95$ & Rhone-Poulenc Rorer Inc. & Fisons PLC & $\$ 2,840$ \\
\hline $01 / 23 / 95$ & Glaxo PLC & Wellcome PLC & $\$ 15,150$ \\
\hline $08 / 29 / 94$ & IVAX Corp. & Zenith Laboratories Inc. & $\$ 594$ \\
\hline $08 / 02 / 94$ & $\begin{array}{l}\text { American Home Products } \\
\text { Corp. }\end{array}$ & American Cyanamid Co. & $\$ 9,700$ \\
\hline $09 / 19 / 91$ & $\begin{array}{l}\text { American Home Products } \\
\text { Corp. }\end{array}$ & Genetics Institute Inc. & $\$ 666$ \\
\hline $07 / 22 / 91$ & Chiron Corp. & Cetus Corp. & $\$ 660$ \\
\hline $07 / 27 / 89$ & Bristol-Myers Co. & Squibb Corp. & $\$ 11,500$ \\
\hline $07 / 17 / 89$ & Dow Chemical Co. & Marion Laboratories Inc. & $\$ 5,700$ \\
\hline $04 / 02 / 89$ & Beecham Group PLC & SmithKline Beckman Corp. & $\$ 7,700$ \\
\hline $02 / 04 / 87$ & $\begin{array}{c}\text { American Home Products } \\
\text { Corp. }\end{array}$ & AH Robins Co. Inc. & $\$ 3,000$ \\
\hline $03 / 03 / 86$ & Schering-Plough Corp. & Key Pharmaceuticals Inc. & $\$ 835$ \\
\hline $07 / 18 / 85$ & Monsanto Co. & GD Searle \& Co. & $\$ 2,700$ \\
\hline
\end{tabular}

Tabella 1

Esempi di fusione considerati nel presente studio (Stime in milioni di dollari)

I guadagni giornalieri delle azioni, relativi al periodo compreso tra i 300 giorni di scambi precedenti e i 45 successivi all'evento sono stati acquisiti dagli archivi CRSP, disponibili alla Austin Graduate School of Business dell'Università del Texas. Il periodo test dello studio è stato definito come $(-45,+45)$, corrispondente approssimativamente ad una finestra temporale comprendente le nove settimane precedenti e le nove settimane successive all'evento. Sulla base di questo periodo test, sono state analizzati specifici periodi: (1) tutti i giorni compresi tra il 45esimo precedente e il 45esimo successivo all'evento, o $(-45,+45)$; (2) un'analisi pre-evento relativa al periodo compreso tra il 45esimo giorno precedente e il giorno dell'evento, con le "periodi" $(-45,-5),(-5,0),(-2,0)$ e $(-1,0)$; e (3) un'analisi post-evento relativa al periodo compreso tra il giorno successivo all'evento e il 45 esimo giorno successivo all'evento, con le periodi $(+1,+2),(+1,+3),(+$ 
$1,+6)$ e $(+6,+45)$. E' stata altresì condotta un'analisi post-hoc per analizzare i profitti degli intervalli(-1, + 1), $(-2,+2)(\mathrm{e}(-5,+5)$. I risultati sono stati riportati come rendimenti anomali (AR) o rendimenti cumulativi ( anomali (CAR) per le singole società; i guadagni del portafoglio azionario sono stati riportati come rendimenti anomali medi (AR) o come rendimenti cumulativi medi (CAR).11 modello ( finanziario dei guadagni del prezzo azionario è ( stato elaborato sulla base del single index market ( model (SIMM). Il SIMM è cosi espresso:

$$
\begin{aligned}
& R_{j t}=a_{j}+b_{j} R_{m t}+\hat{I}_{j t} \\
& \text { dove } R_{i t}=\text { rendimento del titolo } \mathrm{j} \text { nel periodo } \mathrm{t} \text {; } \\
& R_{m t}^{f t}=\text { rendimento dell'indice di mercato nel } \\
& \text { periodo } t \text {; } \\
& a_{j}=\text { costante che determina il valore del } \\
& \text { titolo } \mathrm{j} \text { indipendentemente dal mercato; } \\
& b_{j}=\text { misura del rischio sistematico del tito- } \\
& \text { lo j relativo all'indice di mercato; } \\
& \hat{I}_{j t}=\text { residuale dell'equazione. (15) }
\end{aligned}
$$

Le stime dei parametri SIMM sono state calcolate mediante un metodo OLS (ordinary least squares) sulla base di un periodo di stima compreso tra il 300esimo e il 46esimo giorno precedente la data dell'annuncio. L'indice di mercato (Rmt) utilizzato nell'equazione SIMM è stato definito come l'indice Standard and Poor's 500 (S\&P 500); questi valori sono stati ottenuti dal database CRSP. Nello studio è stata utilizzata la procedura di standardizzazione del guadagno proposta da Patell e resa popolare da Dodd e Warner. Questo metodo, conosciuto come Patell Standardized Residual test (PSR), effettua una correzione per le differenti varianze residuali comuni a tutti i titoli, e si tara sul numero di osservazioni effettuate nel periodo stimato $(16,17)$. Le analisi statistiche sono state elaborate mediante SAS? Release 6.12 e EventusTM Version 6.3 $(18,19)$. il livello alfa di significatività statistica è stato fissato a 0,05 . E' stata impiegata una distribuzione di probabilità a due code poiché non è stata formulata alcuna ipotesi a priori sulla direzione del guadagno.

\section{RISULTATI}

Le stime medie per i parametri beta del SIMM sono state 0,966 e 0,920 per i portafogli delle aziende acquirenti e acquisite, rispettivamente. I rendimenti giornalieri medi durante il periodo di stima sono stati dello $0,00082 \%$ per il portafoglio azionario degli acquisitori e dello $0,00099 \%$ per il portafoglio azionario degli acquisiti. il test statistico di Durbin-Watson per l'autocorrelazione di primo ordine dei residuali ha indicato che né il portafoglio azionario degli

acquisitori, né quello degli acquisiti possedevano una correlazione seriale significativa al livello 0,05 . Analisi Giornaliera

La ricerca precedente sulle fusioni ed acquisizioni indicava uniformemente che i guadagni maggiori e più significativi avvengono il giorno del primo annuncio pubblico. La tabella 2 riassume i rendimenti dei titoli delle società acquirenti, delle società acquisite e dei due insiemi (quello degli acquisiti e quello degli acquisitori) nei giorni $-1,0, \mathrm{e}+1$. La figura 1 mostra una rappresentazione grafica dei guadagni per il periodo $(-45,+45)$. 


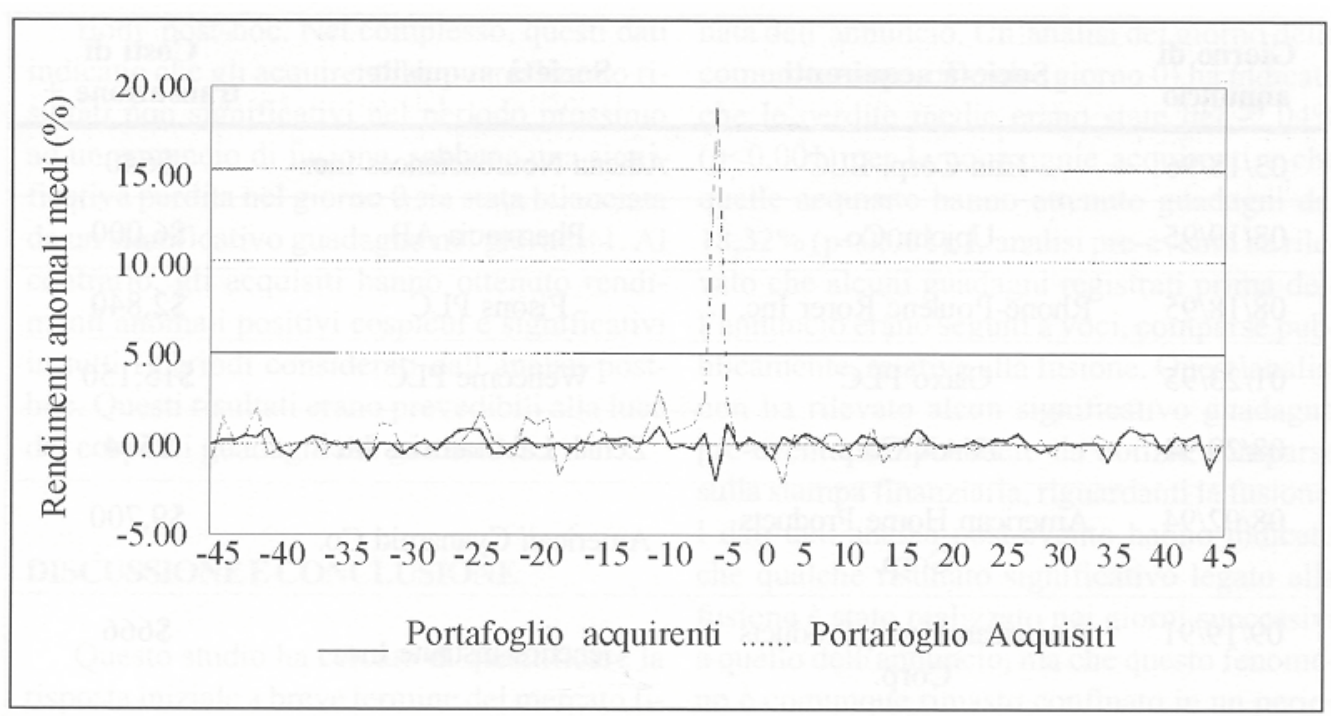

Figura 1

Rendimenti anomali medi del portafoglio delle società acquirenti e acquisite nella finestra temporale considerata

\section{Plusvalenze del giorno 0}

I risultati di questo studio hanno indicato che, nel complesso, gli acquisitori hanno subito perdite significative il giorno dell'annuncio $(A R=-2,04 \%, \mathrm{p}<0.001)$. Quattro aziende acquirenti hanno ottenuto plusvalenze, 10 società acquirenti hanno ottenuto rendimenti negativi; questa proporzione non è risultata significativamente diversa con un $\mathrm{Z}$ test generalizzato (generalized sign $\mathrm{Z}$ test). I cinque maggiori acquirenti nel giorno 0 hanno realizzato guadagni medi dell'1,57\% ( $<<0,05)$, i quattro intermedi perdite significative, $1,96 \%(\mathrm{p}<0,001)$, ed i cinque acquirenti minori hanno ottenuto un significativo-5,70\% ( $<<0,001)$. In contrasto con le perdite degli acquirenti, la maggior parte delle società acquisite hanno fatto registrare grandi, significativi rendimenti positivi nella giornata dell'annuncio della fusione. Nel complesso, il portafoglio azionario degli acquisiti ha beneficiato di un profitto statisticamente significativo nella giornata dell'annuncio (AR 18,32\%, p<0.001). Undici società acquisite hanno realizzato guadagni, 3 hanno subito perdite; questa proporzione è risultata statisticamente diversa con un $\mathrm{Z}$ test generalizzato al livello di significatività di 0,05 . Le cinque maggiori aziende obiettivo delle acquisizioni hanno ottenuto un profitto, statisticamente significativo, del $42,64 \% \%$ ( $\mathrm{P}<\mathrm{O}, \mathrm{OOl}$ ), i quattro intermedi un significativo $13,15 \%$ ( $<<0,00$ 1), mentre i cinque obiettivi minori un $1,86 \%$, non significativo. Sulla base dei risultati del giorno dell'annuncio, ci sono le evidenze per supportare l'ipotesi che la maggior parte della generazione di ricchezza collegata ad una fusione beneficia gli azionisti della società acquisita. La maggiore fusione nell'industria biotecnologica del 1991, la fusione tra la Chiron Corpo e la Cetus Corpo ha costituito l'unico caso in cui sia l'acquirente, sia la società acquisita hanno subito perdite nella giornata dell' annuncio. In seguito all'annuncio, gli analisti industriali affermarono che l'offerta economica della Chiron superava il valore di Cetus, svalutando di conseguenza il valore del titolo. I portavoce aziendali confermarono che in seguito al completamento della fusione ci sarebbero state perdite a breve termine, ma che queste sarebbero state seguite da guadagni a lungo termine. 


\begin{tabular}{|c|c|c|c|c|c|c|c|}
\hline \multirow{2}{*}{$\begin{array}{c}\text { Società } \\
\text { acquirente }\end{array}$} & \multicolumn{3}{|c|}{ AR acquirente (\%) } & \multirow{2}{*}{$\begin{array}{c}\text { Società } \\
\text { acquisita }\end{array}$} & \multicolumn{3}{|c|}{ AR acquisita (\%) } \\
\hline & Giorno -1 & Giorno 0 & Giorno 1 & & Giorno -1 & Giorno 0 & Giorno 1 \\
\hline $\begin{array}{l}\text { Elan Corp. } \\
\text { PLC }\end{array}$ & 0.46 & $\begin{array}{c}-2.87 \\
*\end{array}$ & $\begin{array}{l}4.28 \\
* * *\end{array}$ & $\begin{array}{c}\text { Athena } \\
\text { Neuroscien- } \\
\text { ces Inc. }\end{array}$ & -0.34 & $\begin{array}{c}11.23 \\
*\end{array}$ & 3.63 \\
\hline Upjohn Co. & $\begin{array}{l}9.24 \\
* * *\end{array}$ & $\begin{array}{c}2.94 \\
*\end{array}$ & $\begin{array}{l}5.28 \\
* * *\end{array}$ & $\begin{array}{c}\text { Pharmacia } \\
\text { AB }\end{array}$ & -2.19 & $\begin{array}{c}0.53 \\
*\end{array}$ & -0.55 \\
\hline $\begin{array}{l}\text { Rhone-Poul- } \\
\text { enc Roer } \\
\text { Inc. }\end{array}$ & $\begin{array}{l}-0.24 \\
* * *\end{array}$ & $\begin{array}{c}-2.93 \\
*\end{array}$ & $\begin{array}{l}4.20 \\
* *\end{array}$ & Fisions PLC & -1.16 & $\begin{array}{c}42.40 \\
* * *\end{array}$ & -1.59 \\
\hline Glaxo PLC & -0.09 & $\begin{array}{l}-7.01 \\
* * *\end{array}$ & 0.60 & $\begin{array}{l}\text { Wellcome } \\
\text { PLC }\end{array}$ & 1.54 & $\begin{array}{l}40.45 \\
* * *\end{array}$ & 0.77 \\
\hline IVAX Corp. & -1.79 & -3.19 & -0.36 & $\begin{array}{c}\text { Zenith } \\
\text { Laboratories } \\
\text { Inc. }\end{array}$ & 3.80 & 3.53 & 1.67 \\
\hline $\begin{array}{l}\text { American } \\
\text { Home } \\
\text { Products } \\
\text { Corp. }\end{array}$ & -0.23 & -1.19 & 0.97 & $\begin{array}{c}\text { American } \\
\text { Cyanamid } \\
\text { Co. }\end{array}$ & $\begin{array}{l}3.42 \\
* *\end{array}$ & $\begin{array}{c}47.66 \\
* * *\end{array}$ & $\begin{array}{l}-2.59 \\
*\end{array}$ \\
\hline $\begin{array}{l}\text { American } \\
\text { Home } \\
\text { Products } \\
\text { Corp. }\end{array}$ & 1.46 & 0.53 & $\begin{array}{c}2.22 \\
*\end{array}$ & $\begin{array}{c}\text { Genetics } \\
\text { Institute Inc. }\end{array}$ & 2.25 & -0.49 & 3.54 \\
\hline $\begin{array}{l}\text { Chiron } \\
\text { Corp. }\end{array}$ & 1.02 & $\begin{array}{l}-9.54 \\
* * *\end{array}$ & $-4.75^{*}$ & Cetus Corp. & 0.31 & $\begin{array}{c}-12.21 \\
* *\end{array}$ & -2.93 \\
\hline $\begin{array}{c}\text { Bristol-My- } \\
\text { ers Co. }\end{array}$ & -0.85 & $\begin{array}{l}-5.82 \\
* * *\end{array}$ & $\begin{array}{l}-2.59 \\
* * *\end{array}$ & $\begin{array}{l}\text { Squibb } \\
\text { Corp. }\end{array}$ & $\begin{array}{c}2.71 \\
* *\end{array}$ & $\begin{array}{c}26.99 \\
* * *\end{array}$ & $\begin{array}{c}-1.98 \\
*\end{array}$ \\
\hline $\begin{array}{c}\text { Dow } \\
\text { Chemical } \\
\text { Co. }\end{array}$ & 0.38 & $\begin{array}{c}-1.87 \\
* *\end{array}$ & $\begin{array}{c}-1.46 \\
*\end{array}$ & $\begin{array}{c}\text { Marion } \\
\text { Laboratories } \\
\text { Inc. }\end{array}$ & -0.78 & $\begin{array}{l}32.34 \\
* * *\end{array}$ & $\begin{array}{c}3.26 \\
*\end{array}$ \\
\hline $\begin{array}{l}\text { Beecham } \\
\text { Group PLC }\end{array}$ & 0.65 & $\begin{array}{l}3.97 \\
* * *\end{array}$ & 0.28 & $\begin{array}{c}\text { SmithKline } \\
\text { Beckman } \\
\text { Corp. }\end{array}$ & $\begin{array}{l}8.67 \\
* * *\end{array}$ & $\begin{array}{l}7.33 \\
* * *\end{array}$ & 0.81 \\
\hline $\begin{array}{l}\text { American } \\
\text { Home } \\
\text { Products } \\
\text { Corp. }\end{array}$ & 0.42 & -1.55 & $\begin{array}{l}2.72 \\
* *\end{array}$ & $\begin{array}{l}\text { A. H. } \\
\text { Robins Co. } \\
\text { Inc. }\end{array}$ & $\begin{array}{l}13.54 \\
* * *\end{array}$ & $\begin{array}{c}50.32 \\
* * *\end{array}$ & $\begin{array}{c}27.06 \\
* * *\end{array}$ \\
\hline $\begin{array}{l}\text { Schering } \\
\text { Plough } \\
\text { Corp. }\end{array}$ & $\begin{array}{c}-3.38 \\
* *\end{array}$ & -1.58 & -0.24 & $\begin{array}{c}\text { Key } \\
\text { Pharmaceut- } \\
\text { icals Inc. }\end{array}$ & -0.11 & -0.67 & 3.13 \\
\hline $\begin{array}{c}\text { Monsanto } \\
\text { Co. }\end{array}$ & -0.39 & 1.59 & $\begin{array}{l}4.41 \\
* * *\end{array}$ & $\begin{array}{l}\text { G. D. Searle } \\
\text { \& Co. }\end{array}$ & 2.66 & $\begin{array}{l}7.01 \\
* * *\end{array}$ & 0.28 \\
\hline $\begin{array}{c}\text { Portfolio AR } \\
\text { I }\end{array}$ & 0.54 & $\begin{array}{c}-2.04 \\
* * *\end{array}$ & $\begin{array}{c}1.11 \\
* *\end{array}$ & & $\begin{array}{r}2.45 \\
* * *\end{array}$ & $\begin{array}{c}18.32 \\
* * *\end{array}$ & $\begin{array}{c}2.47 \\
* *\end{array}$ \\
\hline
\end{tabular}

Tabella 2

Rendimenti Anomali medi (AR) delle società acquirenti, acquisite e dei portafogli nei giorni $-1,0 e+1$. Rendimenti anomali medi $* p<0,05 * * P<0,01 * * * P<0,001$ 


\section{Risultati del giorno -1}

Il portafoglio azionario degli acquisitori, nel complesso, non ha realizzato perdite o guadagni statisticamente significativi il giorno -1 ( $\mathrm{AR}=0,54 \%, \mathrm{NS})$. Sette società acquirenti hanno guadagnato, sette hanno registrato rendimenti negativi; questa proporzione non è risultata significativamente diversa rispetto all'utilizzo di un $\mathrm{Z}$ test generalizzato. Solamente due acquirenti, Upjohn Co. e Schering-Plough Corp., hanno realizzato guadagni o perdite significativi sul titolo il giorno -1, probabilmente in relazione a speculazioni relative alla fusione. Il significativo guadagno del giorno -1 della Upjohn $(\mathrm{AR}=9,24 \%, \mathrm{p}<0.001)$ si è avuto in corrispondenza di voci che la indicavano come potenziale acquisitore di Pharmacia AB (21). La settimana precedente erano circolate voci che affermavano che Pharmacia $\mathrm{AB}$ era coinvolta in trattative di fusione con una società non rivelata, con il risultato che la società obiettivo ottenne rendimenti positivi statisticamente significativi il giomo-4 $(\mathrm{AR}=4,50 \%, \mathrm{p}<\mathrm{O}, \mathrm{Ol})$, il giomo-3 $(\mathrm{AR}=4,38 \%, \mathrm{p}<0,01)$ ed il giomo-2 $(\mathrm{AR}=4,95 \%$, $\mathrm{p}<\mathrm{O}, \mathrm{O}$ 1) (22). Per quanto riguarda la fusione Schering-Plough Corp./Key Pharmaceuticals Inc., si sono registrate perdite significative per la Schering-Plough nel giorno $-1(A R=-3,38 \%, p<0.01)$. Diversi giorni prima la Key Pharmaceuticals Inc. era stata indicata come possibile obiettivo di un'acquisizione ed aveva ottenuto significativi guadagni sul titolo il giorno-5 ( $\mathrm{AR}=9,07 \%, \mathrm{p}<0.001)$, il giorno- $4(\mathrm{AR}=9,43 \%$, $\mathrm{p}<0.001)$, ed il giorno-2 $(\mathrm{AR}=5,13 \%, \mathrm{p}<0.05)(23)$.

L'insieme degli azionisti delle imprese acquisite ha beneficiato di guadagni statisticamente significativi nel giorno $-1(\mathrm{AR}=2,45 \%, \mathrm{p}<0.001)$. Nove società acquisite hanno ottenuto guadagni, 5 subito perdite; questa proporzione non è risultata statisticamente diversa rispetto all'uso di uno $\mathrm{Z}$ test generalizzato. La maggior parte dei risultati significativi del giorno -1 sembra essere stata conseguenza di notizie non correlate alla fusione o acquisizione. American Cyanamid Co., Squibb Corpo e A.H. Robins Co., Inc. annunciarono un rapporto riguardante rispettivamente un assets swap agreement, la politica dei dividendi ed i profitti previsti (24-26). Il significativo rendimento positivo realizzato da SmithKline Beckman Corp., tuttavia, è stato conseguente a voci che la indicavano come potenziale obiettivo; l'acquirente ottenne risultati significativi, collegati alla fusione, il giorno $-3(\mathrm{AR}=3,58 \%, \mathrm{p}<0,05)$ ed il giorno $-1(\mathrm{AR}=8,67 \%, \mathrm{p}<0,001)(27)$.

\section{Risultati del giorno +1}

Il portafoglio azionario degli acquisitori, complessivamente, ha beneficiato di risultati significativamente positivi il giorno $+1(\mathrm{AR}=1.11 \%, \mathrm{p}<0.01)$. Nove società acquirenti hanno ottenuto guadagni, cinque hanno ottenuto rendimenti negativi sul titolo; questa proporzione non è risultata statisticamente diversa utilizzando un $\mathrm{Z}$ test generalizzato. E' notevole che otto società acquirenti abbiano ottenuto profitti significativi il giorno $\mathrm{O}$ e che nove li abbiano ottenuti il giorno +1 ; nel complesso questi risultati indicano che i titoli di molte di queste società hanno ottenuto significativi rendimenti positivi post-evento.

Anche il portafoglio azionario degli acquisiti ha beneficiato di significative tendenze positive il giorno +1 $(\mathrm{AR}=2,47 \%, \mathrm{p}<\mathrm{O}, 01)$. Nove aziende obiettivo hanno ottenuto guadagni, 5 hanno subito perdite; questa proporzione non è risultata statisticamente diversa con un $\mathrm{Z}$ test generalizzato. E' importante notare che si è registrato un consistente guadagno per A.H. RobinsCo. Inc. $(A R=27,06 \%, p<0,001)$, il che ha sostanzialmente influenzato i risultati complessivi del portafoglio azionario. Dopo rimozione di questo risultato dal complesso, l'insieme dei rimanenti acquisiti ha ottenuto un guadagno non significativo dello $0,58 \%$.

\section{Analisi Pre-evento}

Oltre a fornire un esame dei risultati nei giorni prima e durante l'evento, l'analisi pre-evento può essere in grado di identificare una sostanziale anticipazione degli investitori delle negoziazioni di fusione, fughe di 
notizie, voci di mercato o insider trading. Le tabelle 3 e 4 mostrano i rendimenti anomali cumulativi preevento realizzate da ognuna delle società acquirenti ed acquisite comprese nel campione.

\section{Periodo (-45, -5)}

I risultati della periodo $(-45,-5)$ hanno indicato che sia gli acquisitori nel complesso (CAR $=3,72 \%$, NS), sia l'insieme degli acquisiti (CAR $=10,67 \%, \mathrm{NS}$ ) hanno ottenuto risultati normali nel periodo precedente la settimana dell'evento. Tra le società acquirenti, due compagnie hanno realizzato plusvalenze statisticamente significative durante l'intervallo(-45, -5): IVAX Corpo (CAR=37,29\%, $\mathrm{p}<0,05)$ e Dow Chemical Co.

$(\mathrm{CAR}=10,60 \%, \mathrm{p}<0,05)$. L'unica società acquisita a realizzare guadagni significativi durante questo periodo è stata American Cyanamid Co. $(\mathrm{CAR}=15,46 \%, \mathrm{p}<0,05)$. Nel complesso, i risultati sono conformi con quelli delle ricerche precedenti, che indicavano che le società acquirenti e le società acquisite non avevano fatto registrare risultati significativi nelle settimane precedenti l'annuncio di fusione.

\section{Periodi $(-5,0),(-2,0)$ e $(-1,0)$}

Il portafoglio azionario degli acquisitori ha subito perdite cumulative medie di - 1,14\% (NS), $-1,77 \%$ $(\mathrm{P}<0,05)$ e $-1,49 \%(\mathrm{p}<0,05)$ durante i periodi $(-5,0),(-2,0)$ e $(-1,0)$, rispettivamente. In accordo con i riscontri del giorno 0 , molte delle società acquirenti hanno ottenuto risultati non significativi anche nelle "periodi" preevento $(-5,0),(-2,0)$ e $(-1,0)$. Le cospicue e significative perdite di valore fatte registrare in alcune di questi periodi da Glaxo PLC, Chiron Group e Bristol-Mayer Co. sono principalmente dovute ai rendimenti negativi del giorno dell'annuncio, siccome non si sono verificate altre perdite significative nella settimana preannuncio. Come menzionato in precedenza, i significativi guadagni preannuncio rilevati per Upjohn Co. e PharmaciaAB sono occorsi quando nella stampa finanziaria comparvero notizie riguardanti una sospettata negoziazione di fusione $(21,22)$.

Le compagnie acquisite, complessivamente, hanno realizzato cospicui guadagni cumulativi medi, statisticamente significativi di 26,24\% ( $<<0.001), 21,87 \%(\mathrm{p}<0.001)$ e $20,77 \%(\mathrm{p}<0.001)$

nei periodi $(-5,0),(-2,0)$ e $(-1,0)$, rispettivamente. In generale, le società acquisite hanno ottenuto rendimenti positivi significativi in almeno una dei periodi pre-evento $(-5,0),(-2,0)$ e $(-1,0)$. Le sole società acquisite che non hanno realizzato significativi guadagni in alcuna dei periodi preevento sono state Athena Neurosciences Inc., Zenith Laboratories Inc. e Genetic Institute Inc.. E' interessante notare che il giorno O Athena fece registrare guadagni significativi in risposta all'annuncio di fusione $(A R=I 1,23 \%, p<0,05)$; il giorno -5 ottenne un guadagno $(\mathrm{AR}=14,00 \%, \mathrm{p}<\mathrm{O}, \mathrm{Ol})$ in seguito all'approvazione di un farmaco da parte della FDA (28). Zenith Laboratories Inc. non ottenne risultati significativi in alcuno dei giorni compresi tra il-5 e il giorno 0 . Infine, l'unica plusvalenza significativa realizzata da Genetics Institute Inc. durante il periodo $(-5,0) \mathrm{fu}$ conseguenza di una dichiarazione riguardante l'approvazione di un brevetto, il giorno $-5(\mathrm{AR}=8,63 \%$, $\mathrm{p}<0,05)(29)$.

Nel complesso, i risultati dell'analisi pre-evento indicano che gli unici risultati significativi collegati alla fusione fatti registrare prima del giorno 0 si sono verificati in seguito a voci apparse nella stampa specializzata. Le fusioni che confermano questa nozione includono la UpjohnCo./Pharmacia AB, la Schering-Plough Corp./Key Pharmaceuticals Inc., e la Beecham Group PLC/SmithKline Beckman Corpo Due fusioni che non sembrano aver causato guadagni durante la settimana precedente il giorno dell' annuncio, giorno O compreso, sono la IV AX Corp./Zenith Laboratories Inc. e la American Home Products Inc./Genetics Institute Inc. Sebbene American Home Products abbia ottenuto un guadagno nel giorno -4 $(\mathrm{AR}=2,79 \%, \mathrm{p}<\mathrm{O}, \mathrm{O} 1)$ e Genetics Institute abbia ottenuto rendimenti anomali positivi il giorno -5 (AR= $8,63 \%, \mathrm{p}<\mathrm{O}, \mathrm{O} 5)$, in nessuno dei due casi questi risultati sono stati attribuiti a notizie o voci sul loro annuncio di fusione. Si noti come la American Home Products Inc. abbia ottenuto un guadagno significativo il giorno 
$+1(\mathrm{AR}=2,22 \%, \mathrm{p}<\mathrm{O}, \mathrm{O} 5)$, mentre la Genetics Institute Inc. non abbia fatto registrare risultati significativi il giorno successivo all'annuncio ( $\mathrm{AR}=3,54 \%, \mathrm{NS})$.

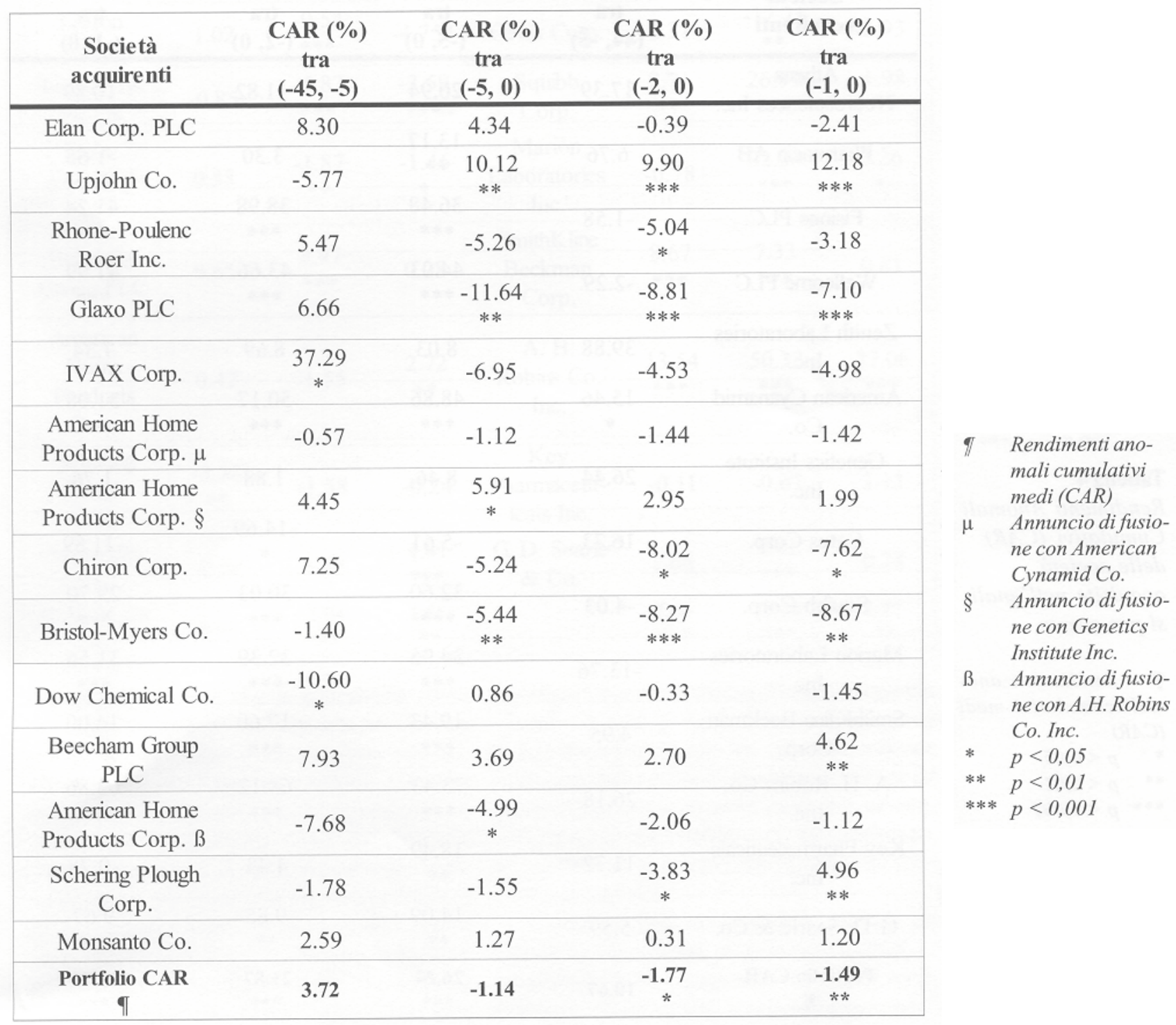

\section{Tabella3}

Rendimenti Anomali Cumulativi (CAR) delle società acquirenti nell'analisi pre-evento.

\section{Analisi Post-evento}

I risultati dell'analisi post-evento possono aiutare a comprendere l'evoluzione delle trattative di fusione, le contese tra vari potenziali acquirenti e i cambiamenti post-annuncio delle sensazioni degli investitori. Le tabelle 5 e 6 riportano le plusvalenze cumulative post-evento ottenute da ognuna delle società acquirenti e acquisite contenute nel campione. 


\begin{tabular}{|c|c|c|c|c|c|}
\hline $\begin{array}{l}\text { Società } \\
\text { acquirenti }\end{array}$ & $\begin{array}{c}\text { CAR }(\%) \\
\text { tra } \\
(44,-5)\end{array}$ & $\begin{array}{c}\text { CAR }(\%) \\
\text { tra } \\
(-5,0)\end{array}$ & $\begin{array}{c}\text { CAR }(\%) \\
\text { tra } \\
(-2,0)\end{array}$ & $\begin{array}{c}\text { CAR }(\%) \\
\text { tra } \\
(-1,0)\end{array}$ & \multirow{16}{*}{$\begin{array}{l}\text { I Rendimenti ano- } \\
\text { mali cumulativi medi } \\
\text { (CAR) } \\
* \quad p<0,05 \\
* * \quad p<0,01 \\
* * * \quad p<0,001\end{array}$} \\
\hline $\begin{array}{c}\text { Athena } \\
\text { Neurosciences Inc. }\end{array}$ & 17.39 & 20.94 & 11.82 & 10.89 & \\
\hline Pharmacia $\mathrm{AB}$ & 6.76 & $\begin{array}{c}13.17 \\
* *\end{array}$ & 3.30 & -1.65 & \\
\hline Fisions PLC & -1.58 & $\begin{array}{l}36.48 \\
* * *\end{array}$ & $\begin{array}{c}38.98 \\
* * *\end{array}$ & $\begin{array}{l}41.24 \\
* * *\end{array}$ & \\
\hline Wellcome PLC & -2.29 & $\begin{array}{l}44.03 \\
* * *\end{array}$ & $\begin{array}{c}43.66 \\
* * *\end{array}$ & $\begin{array}{c}41.99 \\
* * *\end{array}$ & \\
\hline $\begin{array}{l}\text { Zenith Laboratories } \\
\text { Inc. }\end{array}$ & 39.88 & 8.03 & 8.69 & 7.34 & \\
\hline $\begin{array}{c}\text { American Cyanamid } \\
\text { Co. }\end{array}$ & $\begin{array}{c}15.46 \\
*\end{array}$ & $\begin{array}{c}48.86 \\
* * *\end{array}$ & $\begin{array}{c}50.17 \\
* * *\end{array}$ & $\begin{array}{c}51.08 \\
* * *\end{array}$ & \\
\hline $\begin{array}{l}\text { Genetics Institute } \\
\text { Inc. }\end{array}$ & 26.44 & 8.46 & 1.88 & 1.76 & \\
\hline Cetus Corp. & 16.73 & -5.61 & $\begin{array}{c}-14.69 \\
*\end{array}$ & -11.89 & \\
\hline Squibb Corp. & -4.03 & $\begin{array}{l}32.60 \\
* * *\end{array}$ & $\begin{array}{l}30.03 \\
* * *\end{array}$ & $\begin{array}{l}29.70 \\
* * *\end{array}$ & \\
\hline $\begin{array}{l}\text { Marion Laboratories } \\
\text { Inc. }\end{array}$ & -13.76 & $\begin{array}{l}34.95 \\
* * *\end{array}$ & $\begin{array}{l}32.39 \\
* * *\end{array}$ & $\begin{array}{l}31.56 \\
* * *\end{array}$ & \\
\hline $\begin{array}{l}\text { SmithKline Beckman } \\
\text { Corp. }\end{array}$ & 4.95 & $\begin{array}{c}19.43 \\
* * *\end{array}$ & $\begin{array}{l}17.60 \\
* * *\end{array}$ & $\begin{array}{c}16.00 \\
* * *\end{array}$ & \\
\hline $\begin{array}{l}\text { A. H. Robins Co. } \\
\text { Inc. }\end{array}$ & 26.18 & $\begin{array}{l}73.43 \\
* * *\end{array}$ & $\begin{array}{c}68.12 \\
* * *\end{array}$ & $\begin{array}{l}63.86 \\
* * *\end{array}$ & \\
\hline $\begin{array}{l}\text { Key Pharmaceuticals } \\
\text { Inc. }\end{array}$ & 11.72 & $\begin{array}{c}18.49 \\
* *\end{array}$ & 4.43 & -0.78 & \\
\hline G. D. Searle \& Co. & 5.59 & $\begin{array}{c}14.09 \\
* *\end{array}$ & $\begin{array}{l}9.85 \\
* *\end{array}$ & $\begin{array}{l}9.67 \\
* * *\end{array}$ & \\
\hline $\begin{array}{c}\text { Portfolio CAR } \\
\text { ๆ }\end{array}$ & 10.67 & $\begin{array}{c}26.24 \\
* * *\end{array}$ & $\begin{array}{c}21.87 \\
* * *\end{array}$ & $\begin{array}{c}20.77 \\
* * *\end{array}$ & \\
\hline
\end{tabular}

Tabella 4

Rendimenti Anomali Cumulativi (CAR) delle società acquisite nell'analisi pre-evento

\section{Periodi $(+1,+2)$, $(+1,+3)$ e $(+1,+6)$}

Nel periodo compreso dagli intervalli $(+1,+2),(+1,+3)$ e $(+1,+6)$, l'insieme degli acquirenti ha ottenuto rendimenti positivi cumulati medi di $0,98 \%$ (NS), $1,29 \%$ (p<0,05) e $0,93 \%$ (NS), rispettivamente. Il portafoglio azionario degli acquisiti non ha ottenuto perdite o guadagni significativi nel periodo compreso nei periodi $(+1,+2),(+1,+3)$ e $(+1,+6)$; i guadagni cumulativi medi sono stati $2,08 \%(\mathrm{NS}), 2,53 \%$ (NS) e $0,00 \%$ (NS), rispettivamente.

Sebbene otto società acquirenti abbiano ottenuto guadagni significativi il giorno +1 , solamente quattro hanno fatto registrare rendimenti positivi in uno degli intervalli $(+1,+2),(+1,+3)$ e $(+1,+6)$ : Elan Corpo PLC,

Bristol-Myers Co., Dow Chemical Co. e Monsanto Co. Nell'intervallo $(+1,+6)$ l'unico guadagno significativo fatto registrare da Elan fu quello del giorno $+1(\mathrm{AR}=4,28 \%, \mathrm{p}<\mathrm{O}, \mathrm{Ol})$. Bristol-Myers è stato l'unico 
acquirente ad ottenere risultati significativi, ma negativi, in tutti i periodi: la compagnia ottenne rendimenti negativi significativi il giorno $+1(A R=-2,59 \%, \mathrm{p}<\mathrm{O}, \mathrm{OO})$ e il giorno $+5(\mathrm{AR}=-1,69 \%, \mathrm{p}<\mathrm{O}, \mathrm{O} 5)$. Dow

Chemical fece registrare risultati significativi il giorno $+1(\mathrm{AR}=-1,46 \%, \mathrm{p}<\mathrm{O}, \mathrm{O} 5)$, il giomo $+2(\mathrm{AR}=1,76 \%$, $\mathrm{p}<0,01)$ e il giorno $+3(\mathrm{AR}=1,99 \%, \mathrm{p}<0,01)$. Il risultato del giorno +3 seguì ad una relazione su forti profitti (31). Monsanto, infine, fece registrare guadagni significativi il giorno $+1(A R=4,41 \%, p<0.001)$, il giorno $+3(\mathrm{AR}=3,66 \%, \mathrm{p}<0.01)$ eil giorno $+4(\mathrm{AR}=-2,28 \%, \mathrm{p}<0.01)$. Il giorno +3 fu pubblicata una relazione sui guadagni (32).

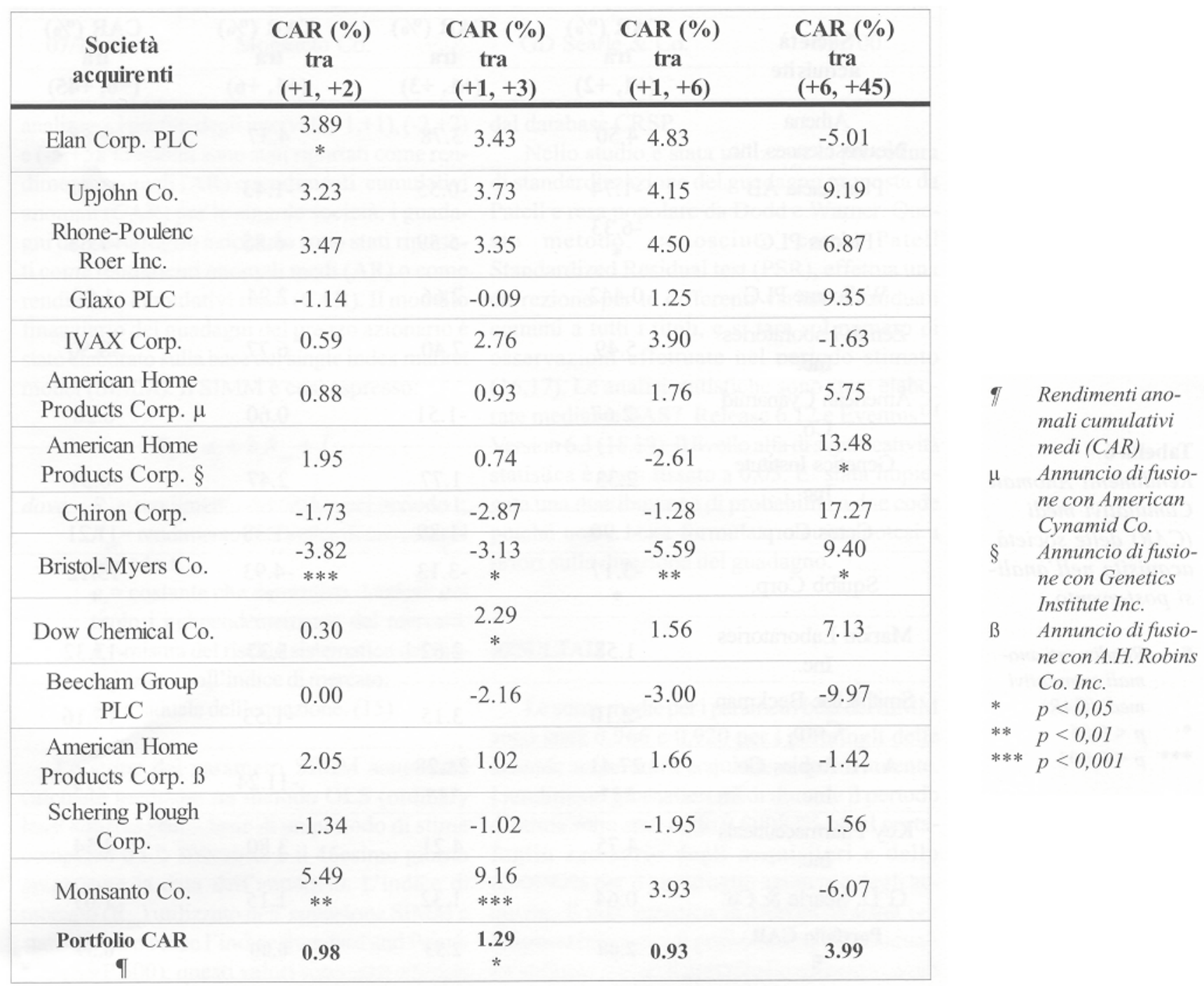

Tabella 5

Rendimenti Anomali Cumulativi medi (CAR) delle società acquirenti nell'analisi post-evento

Tra le società acquisite, tre ottennero risultati significativi in almeno uno degli intervallii $(+1,+2),(+1,+3)$ o $(+$ $1,+6)$ : FisonsPLC, Squibb Corpo e A.H. Robins Co., Inc. L'unico guadagno significativo fatto registrare da Fisons nel periodo $(+1,+6)$ fu quello realizzato nel giorno $+2(\mathrm{AR}=-4,73, \mathrm{p}<0.05)$, in seguito al fallimento 
di alcune controfferte da parte di società che cercavano di contrastare l'offerta di acquisizione della RhonePoulenc (33). Sebbene Squibb Corpo sia stata l'unica società che abbia ottenuto risultati significativi, quantunque negativi, in ognuno dei tre intervalli, la sola minusvalenza significativa fatta registrare durante l'intervallo $(+1,+6)$ è quella del giorno $+1(\mathrm{AR}=-1,98, \mathrm{p}<0,05)$; l'azienda ottenne risultati prevalentemente negativi, anche se non significativi, fino al giorno +5 . Come accennato in precedenza, il giorno +1 A.H. Robins Corp., Inc. fece registrare cospicui rendimenti positivi post-evento ( $A R=27,06, p<O, O O I$ ), che influenzarono sostanzialmente il risultato complessivo del gruppo delle società acquisite. E' da sottolineare il fatto che il giorno +6 American Home Products ritirò la sua offerta d'acquisizione, causando una perdita significativa per A.H. Robins (AR=-29,03, p<0.001). In seguito ad ulteriori trattative, Robins fu acquisita da American Home Products nel 1989.

\section{Periodo $(+6,+45)$}

Per completare l'analisi post-evento, rimane da segnalare che nessun risultato significativo è stato fatto registrare durante l'intervallo $(+6,+45)$ né dall'insieme degli acquirenti, né da quello degli acquisiti. Gli acquirenti ottennero un guadagno cumulativo medio del 3,99\% (NS) mentre gli acquisiti dello 0,59\% (NS). Sulla base di questi risultati, sembra che i guadagni post-evento significativi siano rimasti confinati ad un periodo relativamente breve, immediatamente successivo al giorno dell'annuncio. Tra le aziende acquirenti, solo American Home Products (in seguito all'annuncio di fusione con Genetics Institute Inc.) e Bristol-Myers Co. ottennero rendimenti positivi significativi. American Home Products ha realizzato un guadagno cumulativo del 13,48\% ( $\mathrm{p}<0,05)$ e Bristol-Myers del 9,40\% ( $\mathrm{p}<0,05)$. Tra le aziende acquisite, Squibb Corpo è stata l'unica compagnia a realizzare risultati significativi durante l'intervallo $(+6,+45):(C A R=13,12 \%$ $\mathrm{p}<0,05)$.

\section{Analisi Post-hoc}

I test relativi alle periodi $(-1,+1),(-2,+2)$ e $(-5,+5)$ possono fornire un'analisi complessiva, ma a breve termine, dell'impatto dell'annuncio di fusione. I risultati di un'analisi post-hoc sono riportati in tabella 7. L'insieme degli acquirenti non ha realizzato alcun risultato significativo nell'analisi dei periodi post-hoc. Nel complesso, questi dati indicano che gli acquirenti hanno realizzato risultati non significativi nel periodo prossimo ad un annuncio di fusione, sebbene una significativa perdita nel giorno $\mathrm{O}$ sia stata bilanciata da un significativo guadagno nel giorno +1 . Al contrario, gli acquisiti hanno ottenuto rendimenti anomali positivi cospicui e significativi in tutti i periodi considerati dall'analisi post- hoc. Questi risultati erano prevedibili alla luce dei cospicui guadagni del giorno 0 .

\section{DISCUSSIONE E CONCLUSIONE}

Questo studio ha cercato di quantificare la risposta iniziale a breve termine del mercato finanziario ad alcune tra le maggiori fusioni orizzontali avvenute nell'ambito dell'industria farmaceutica tra il 1985 e il 1996. I risultati di questo studio supportano le conclusioni della ricerca precedente, che indicava che la maggior parte della ricchezza generata da una fusione veniva beneficiata dagli azionisti della società acquisita, e che tali rendimenti positivi dei titoli erano realizzati prevalentemente durante la giornata dell'annuncio. Un'analisi del giorno della comunicazione ufficiale (giorno 0 ) ha indicato che le perdite medie erano state del $-2,04 \%(\mathrm{p}<0,001)$ per le compagnie acquirenti e che quelle acquisite hanno ottenuto guadagni del 18,32\% $(\mathrm{p}<0,001)$. L'analisi pre-evento ha rilevato che alcuni guadagni registrati prima dell'annuncio erano seguiti a voci, comparse pubblicamente, relative alla fusione. Quest'analisi non ha rilevato alcun significativo 
guadagno pre-evento indipendente da notizie comparse sulla stampa finanziaria, riguardanti la fusione. I dati dell'analisi post-evento hanno indicato che qualche risultato significativo legato alla fusione è stato realizzato nei giorni successivi a quello dell'annuncio, ma che questo fenomeno è comunque rimasto confinato in un periodo di tempo molto ristretto.

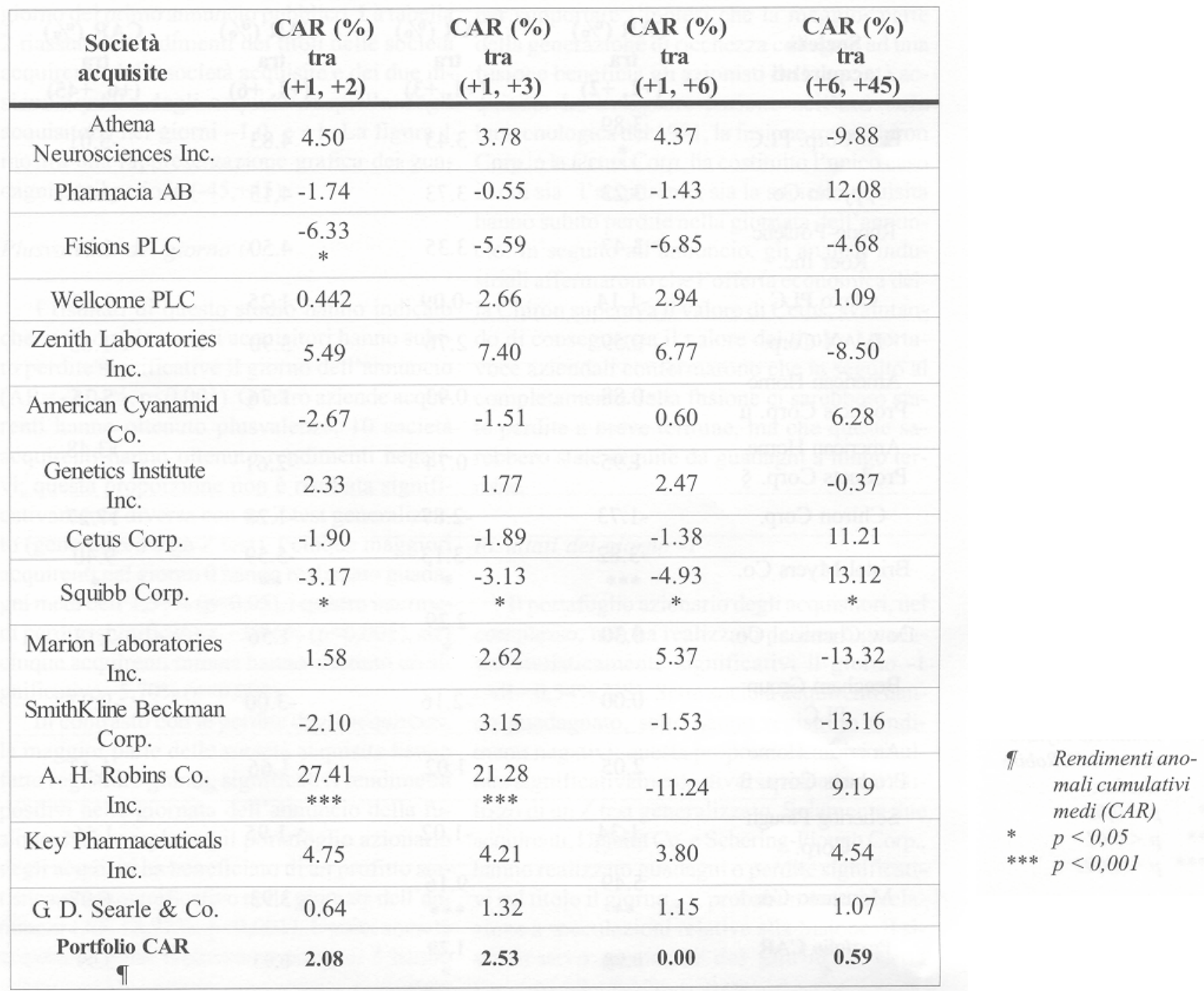

Tabella 6

Rendimenti Anomali Cumulativi medi (CAR) delle società acquisite nell'analisi post-evento

Un'analisi post- hoc ha mostrato che gli acquirenti, presi nel complesso, hanno ottenuto risultati non significativi $(\mathrm{CAR}=-0,38 \%$, NS) durante l'intervallo $(-1,+1)$; una significativa perdita al giorno 0 ( $\mathrm{AR}=-$ $2,04 \%, \mathrm{p}<0.001)$ è stata seguita da un significativo guadagno nel giorno $+1(\mathrm{AR}=1.11 \%, \mathrm{p}<0.01)$. Le aziende acquisite, al contrario, hanno ottenuto, nell'insieme, considerevoli e significativi guadagni durante l'intervallo $(-1,+1)(\mathrm{CAR}=23,23 \%, \mathrm{p}<0.001)$. 
Nonostante l'evidenza empirica documenti una distribuzione ineguale dei rendimenti di una fusione tra gli azionisti delle società acquirenti e di quelle acquisite, le operazioni di fusione ed acquisizione sono generalmente considerate strumento per aumentare il valore delle società nel complesso (35). L'ineguale distribuzione della ricchezza tra società acquirenti e acquisite durante le operazioni M\&A può essere spiegata da una caratteristica delle operazioni di fusione e acquisizione; il fatto, cioè, che spesso gli acquirenti pagano un premio per comprare azioni dagli azionisti della società acquisita. Inoltre, anche la teoria finanziaria sostiene che in caso di perfetta competizione di mercato, la società acquirente dovrebbe realizzare guadagni prossimi a 0 .

Molte delle fusioni considerate da questo studio sembrano supportare queste supposizioni, indicando che la maggior parte dei guadagni legati alla fusione sono stati ottenuti dalle società acquisite e che i risultati realizzati da molte società acquirenti sono sembrati pressoché normali. Sebbene la ricerca indichi che tipicamente le società acquirenti realizzano guadagni pressoché normali in seguito all'annuncio di una fusione, $i$ vantaggi in termini di sinergia, diversificazione, maggior potere di mercato e incentivi fiscali possono fare in modo che le fusioni e le acquisizioni continuino ad essere investimenti interessanti per molti gruppi.

\begin{tabular}{|c|c|c|c|c|c|c|}
\hline \multirow{2}{*}{$\begin{array}{l}\text { Finestra } \\
\text { temporale }\end{array}$} & \multicolumn{2}{|c|}{ Poartafoglio acquirente } & \multicolumn{2}{|c|}{ Portfoglio acquisita } & \multirow{4}{*}{9} & \multirow{5}{*}{$\begin{array}{l}\text { Rendimentiano- } \\
\text { mali cumulativi } \\
\text { medi }(C A R) \\
* \quad p<0,001\end{array}$} \\
\hline & CAR \ (\%) & Z-Statistic & CAR \ $(\%)$ & Z-Statis tic & & \\
\hline$(-1,+1)$ & -0.38 & -0.50 & $23.23 * * *$ & 26.76 & & \\
\hline$(-2,+2)$ & -0.78 & -0.88 & $23.95 * * *$ & 21.01 & & \\
\hline$(-5,+5)$ & -0.61 & -0.44 & $28.42 * * *$ & 16.30 & $* * *$ & \\
\hline
\end{tabular}

Tabella 7

Riepilogo del portafoglio delle società acquirenti e acquisite nell'analisi post-hoc, secondo la finestra temporale considerata.

I risultati di questo studio non dovrebbero essere generalizzati al di là del campione preso in considerazione, perché ognuna delle società e delle fusioni ha presentato caratteristiche e dettagli unici, che renderebbero inattendibili tali generalizzazioni. Sebbene non sia stato adottato nessun metodo di controllo per le caratteristiche specifiche di ogni fusione, va sottolineato che molti fattori sono in grado di influenzare la risposta del mercato finanziario (p.e. le sensazioni degli investitori, l'anticipazione del mercato, il premio pagato, il metodo di pagamento e altre circostanze particolari). Poiché in questo studio non è stato fatto alcun tentativo di misurare la performance complessiva della corporazione, non si possono nemmeno trarre conclusioni sui guadagni o le perdite complessive della corporazione. Inoltre, siccome non è stata inclusa una dettagliata analisi post-integrazione, non dovrebbe venir intrapreso alcun tentativo di previsione del successo o del fallimento di una fusione basandosi unicamente su questi risultati.

Il giorno-evento di questo studio è stato definito come quello in cui la stampa finanziaria ha riportato un annuncio pubblico di trattative di fusione tra gli attori finali della contrattazione; per la definizione della data-evento sono state quindi usate solo le rivelazioni volontarie fatte dalle aziende coinvolte. In questo contesto non sono state considerate voci, fughe di notizie o anticipazioni del mercato. Nei casi di gare tra più acquirenti, l' acquisitore finale può non essere stato quello che aveva iniziato le trattative di fusione. La ricerca futura potrebbe essere rivolta allo studio di un campione più rappresentativo del complesso delle fusioni nell'ambito dell'industria farmaceutica. Un'analisi a lungo termine delle società acquirenti ed 
acquisite che includa misure addizionali di performance economica potrebbe rivelarsi utile alla comprensione degli effetti pre e post-integrazione della fusione. Inoltre, potrebbe rivelarsi utile un confronto tra fusioni attuate con strategie corporative diverse, quali disinvestimento, le alleanze strategiche, gli impegni di co-marketing e le joint ventures.

\section{BIBLIOGRAFIA}

1. Mergerstat Review. Houlihan, Lokey, Howard, and Zukin, LosAngeles, 1986-1996.

2. Tanouye E. Value of some drug firms' acquisitions is questioned. Wall Street J 1996 Nov 19:B4.

3. Jensen MC, Ruback RS. The market for corporate control: the scientific evidence. J Financ Econ 1983;11:5-50.

4. Jarrell GA, Brickley JA, Netter JM. The market for corporate control: the empirica evidence since 1980. J Econ Perspect 1988;2:49-68.

5. Jarrell GA, Poulsen AB. The returns to acquiring firms in tender offers: evidence from three decades. Financ Manage I 989; (Autumn): 12-19.

6. Brown SJ, Warner JB. Measuring security price performance. J Financ Econ 1980;8:205-58.

7. Brown SJ, Warner JB. Using daily stock returns: the case of event studies. J Financ Econ 1985;14:3-31.

8. Dyckman T, Philbrick D, Stephan J. A comparison of event study methodologies using daily stock returns: a simulation approach. J Account Res 1984;22: 1-33.

9. Bowman RG Understanding and conducting event studies. J Bus Finance Account 1983; 10:561-84.

10. Peterson PP. Event studies: a review of issues and methodology. Q J Bus Econ 1989;28:36-66.

11. Henderson GV. Problems and solutions in conducting event studies. J Risk In-sur 1990;57:282-306.

12. Strong N. Modeling abnormal returns: a review article. J Bus FinanceAc-count 1992;19:533-53.

13. MacKinIay AC. Event studies in economics and finance. JEconLit 1997;35:13-39.

14. Issues of Mergers \& Acquisitions from 1986 to 1996.

15. Fama EF. Foundations of finance. New York: Basic Books; 1976:63-132.

16. Patell JM. Corporate forecasts of earnings per share and stock price behavior: empirical tests. J Account Res 1976;14:246-76.

17. Dodd P, Warner JB. On corporate governance: a study of proxy contests. JFinanc Econ 1983;11:401-38

18. SAS/STAT users guide, version 6. Cary, NC: SAS Institute; 1997.

19. Cowan AR. Eventus version 6.3. Ames, 1A: CowanResearch, L.C.; 1997.

20. Anonymous. Cetus, Chiron set biggest U.S. biotech merger. Reuter Bus Rep 1991 JuI 22.

21. Genevieve J. Blue-chip stocks drift lower; NASDAQ edges higher: Reuter Bus Rep 1995 Aug 18.

22. Anonymous. Swedish shares track global slide downwards. Reuter Eur Bus Rep 1995 Aug 14.

23. Anonymous. Dow edges a little nearer to 1,700. N Y Times 1986 Feb 25:D10.

24. Anonymous. London shares higher in cautious early trade. AFX News 1994 Aug 1.

25. Anonymous. Squibb declares quarterly dividend. Bus Wire 1989 JuI 26.

26. Anonymous. A. H. Robins reports increased sales and earnings. PR Newswire 1987 Feb 3.

27. Anonymous. SmithKline stock rises on report of possible merger. Reuter Bus Rep 1989 Mar 29.

28. Anonymous. Athena says receives FDA approval letter. Reuter Financ Service 1996 Mar 11 :Financial Report Section.

29. Anonymous. Genetics Institute licensee, Sandoz receives European patent for GM-CSF. PR Newswire 1991 Sept 11: Financial News Section.

30. Anonymous. American Home Products and Eisai Company sign joint agreement. PR Newswire 1991 Sept 11 :Financial News Section.

31. Anonymous. Dow announces second-quarter sales and earnings records. PR Newswire 1989 Jul 20.

32. Anonymous. Upturn on economic optimism. Financ Times 1985 JuI 24. 
Farmeconomia e percorsi terapeutici 2001; 2 (3)

33. Anonymous. London shares at midsession. AFX News 1995 Aug 22.

34. Anonymous. American Home Products withdraws acquisition offer. PR Newswire 1987 Feb 12.

35. Weston JF, Chung KS, Siu JA. Takeovers, restructuring, and corporate governance. Upper Saddie River, NJ: PrenticeHalI,Inc.; 1998:124-45. 